\title{
Relación entre la composición corporal y sintomatología en mujeres de Aguascalientes que padecen fibromialgia
}

\author{
Relationship between body composition and \\ symptoms in women with fibromyalgia from \\ Aguascalientes
}

\author{
Esparza-Santillán, Alma Karina; Hernández-Ramírez, Andrea \\ Mariana; Muñoz-Hermosillo, Denniss Jaquelin; Ramírez-Orozco, \\ Ricardo Ernesto
}

\begin{abstract}
Alma Karina Esparza-Santillán
Universidad Autónoma de Aguascalientes, México

Andrea Mariana Hernández-Ramírez

Universidad Autónoma de Aguascalientes, México

Denniss Jaquelin Muñoz-Hermosillo

Universidad Autónoma de Aguascalientes, México

Ricardo Ernesto Ramírez-Orozco

dcmrero@gmail.com

Universidad Autónoma de Aguascalientes, México
\end{abstract}

Lux Médica

Universidad Autónoma de Aguascalientes, México

ISSN: 2007-1655

Periodicidad: Cuatrimestral

vol. 16, núm. 48, 2021

luxmedica.editorial@gmail.com

Recepción: 05 Febrero 2020

Aprobación: 31 Mayo 2021

URL:

https://revistas.uaa.mx/index.php/luxmedica/article/view/3269

Autor de correspondencia:dcmrero@gmail.com

Poítica de acceso abierto la Revista Lux Médica proporciona un acceso abierto a su contenido, basado en el principio de que ofrecer un acceso libre a las investigaciones ayuda a incrementar el intercambio global del conocimiento. La LM no cobra ni cobrará ningún cargo a sus lectores por concepto de suscripción, ni a los autores por enviar, procesar o publicar sus artículos. Como condición de publicación, los autores acuerdan liberar sus derechos de autor bajo una licencia compartida, específicamente la licencia de Creative Commons Reconocimiento-NoComercial-Compartir Igual 4.0 Internacional Esta licencia permite a cualquier persona compartir, copiar y redistribuir el material en cualquier medio o formato bajo los siguientes términos: - Dar crédito al autor del texto - No hace uso del material con propósitos comerciales - No transformar o modificar el material. Los autores ceden el derecho de la primera publicación a esta revista, pero conservarn sus derechos de autor, de tal forma que pueden realizar otros acuerdos contractuales
Resumen: Introducción: La fibromialgia es una enfermedad con diversas complicaciones que afectan al estado nutricional. Niveles de composición corporal alterados (exceso de adiposidad o sarcopenia) son condicionantes para el control y la aparición de manifestaciones clínicas. Objetivo: Describir la asociación de la composición corporal y la sintomatología de la fibromialgia. Materiales y métodos: Estudio descriptivo transversal en el que participaron 27 mujeres con fibromialgia de Aguascalientes, México. Se realizó el cuestionario sobre los efectos de la fibromialgia (FIQ) para recolectar datos de sintomatología; para la composición corporal se utilizó una báscula de control corporal OMRON HBF-514C, analizando peso, masa grasa, masa

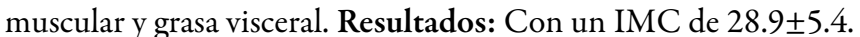
Se clasificó en desnutrición, normopeso, sobrepeso y obesidad (3.7\%, 25.9\%, 29.6\% y 40.7\% respectivamente). El 66.7\% de las pacientes presentó un porcentaje muy elevado de grasa, el 22.9\% obtuvo porcentaje elevado y solo el $11.1 \%$ presentó un porcentaje normal para su edad. No hubo diferencias entre un porcentaje de grasa normal, elevado y muy elevado, ni en el IMC en relación con el puntaje total del FIQ. La relación entre la composición corporal y las variables clínicas obtenidas en el FIQ demostraron que no hay relación significativa. Conclusiones: No hubo relación significativa entre la composición corporal y la sintomatología de fibromialgia; sin embargo, existe evidencia que demuestra lo contrario. La prevalencia de obesidad y sobrepeso es alta en pacientes con fibromialgia, es importante continuar estudios para establecer la relación que existe entre estas condiciones, generando a futuro un adecuado tratamiento integral.

Palabras clave: Composición corporal, Fibromialgia, FIQ, Obesidad.

Abstract: Introduction: Fibromyalgia is a disease with several complications that affect nutritional status. Altered body composition levels (high adiposity or sarcopenia) are conditioning factors for the control and appearance of clinical manifestations. Objective: To describe the association of body composition and 
independientes y adicionales para la distribución no exclusiva de la versión del artículo publicado en esta revista ( por ejemplo, incluirlo en un repositorio institucional o publicarlo en un libro) siempre que indiquen claramente que el trabajo se publicó por primera vez en ésta.

\section{(i)(2)}

Esta obra está bajo una Licencia Creative Commons AtribuciónNoComercial-CompartirIgual 4.0 Internacional.

Cómo citar este artículo: Esparza Santillán , A. K., Hernández Ramírez, A. M., Muñoz Hermosillo, D. J., Ramírez Orozco, R. E. Relación entre la composición corporal y sintomatología en mujeres de Aguascalientes que padecen fibromialgia. Lux Médica, 16(48). Recuperado a partir de https://revistas.uaa.mx/index.php/luxmedica /article/view/3269 fibromyalgia symptoms. Materials and Methods: Descriptive cross-sectional study, volunteers: 27 women with fibromyalgia from Aguascalientes, Mexico. Fibromyalgia impact questionnaire (FIQ) was applied to collect data on symptoms; For body composition, a body control scale (OMRON HBF-514C) was used to analyze weight, fat mass, muscle mass, and visceral fat. Results: volunteers presented a BMI of $28.9 \pm 5.4$. Malnutrition, normal-weight, overweight, and obesity rates were $3.7 \%, 25.9 \%$, $29.6 \%$, and $40.7 \%$, respectively. $66.7 \%$ of patients presented an extremely increased percentage of fat, $22.9 \%$ obtained a high percentage, only $11.1 \%$ presented healthy levels for her age. Normal, high, and very high-fat percentages, nor in BMI concerning total FIQ score were different. Relationship between body composition and clinical variables obtained in the FIQ showed that there is no significant association. Conclusions: There was no significant relationship between body composition and fibromyalgia symptoms. However, there is evidence to show otherwise. The prevalence of obesity and overweight is high in patients with fibromyalgia being important to continue studies to establish the relationship between these conditions, generating an adequate comprehensive treatment in the future.

Keywords: Body composition, Fibromyalgia, FIQ, Obesity.

\section{INTRODUCCIÓN}

La fibromialgia (FM) es una enfermedad reumatológica reconocida por la Organización Mundial de la Salud (OMS) e incluida en la Clasificación Internacional de Enfermedades en 1992. Dicho padecimiento afecta a entre el 2 y el $5 \%$ de la población mundial. ${ }^{1}$

El American College of Rheumatology (ACR) ha definido a la FM como una "enfermedad neurológica que ocasiona dolor crónico musculoesquelético". Se caracteriza por la presencia de debilidad muscular, alteración del sueño, depresión, problemas de memoria, problemas de atención, entre otros. ${ }^{2}$

El diagnóstico de la enfermedad debe realizarlo un médico especialista mediante una prueba de sensibilidad al dolor en 19 partes del cuerpo. Además, se evalúa la presencia de dolor general, síntomas como cansancio, y problemas de memoria y atención. Los síntomas deben estar presentes de forma constante y con la misma intensidad durante tres meses antes de la fecha del diagnóstico. ${ }^{2}$

En la actualidad, se desconoce el factor causante que explique los mecanismos fisiopatológicos de la fibromialgia. En algunos estudios se sugiere que la fibromialgia deriva de una alteración de las vías de conducción y control del dolor, mientras que en otros casos se cree que el desencadenante de dicho dolor provendría de un trauma psicológico. ${ }^{3}$

Entre las causas más estudiadas se encuentran los mecanismos de sensibilización central, los cuales generan una amplificación de la señal nociceptiva. Otro de los mecanismos son los procesos de neuroplasticidad en donde se encuentran implicadas estructuras como los receptores de neurocininas 1 (sustancia P) y células gliales responsables de la secreción de factores neutróficos y citocinas proinflamatorias. ${ }^{3}$ 
México se posicionó en el octavo lugar a nivel mundial de prevalencia de fibromialgia, con un $0.68 \%$, siendo las mujeres el grupo mayormente afectado. ${ }^{4}$ Por su parte, un estudio demostró que la prevalencia de obesidad en pacientes con fibromialgia es del $32 \%$ al $50 \%$ y la prevalencia de sobrepeso del $21 \%$ al $30 \%{ }^{5,6,7}$

Se sabe que la obesidad es una enfermedad crónica de origen multifactorial. Dicho padecimiento es el resultado del desequilibrio entre la ingesta de energía y el gasto calórico, lo cual se traduce en una acumulación de tejido adiposo. Se conoce que el exceso de tejido adiposo genera un desequilibrio en la homeostasis del organismo, provocando la activación de respuesta del sistema inmune. ${ }^{8,9} \mathrm{La}$ IL-6 y el TNF- $\alpha$ son las citocinas con mayor participación en el proceso de inflamación de la obesidad. ${ }^{10}$

Una investigación reciente en pacientes con fibromialgia describió un incremento en los niveles séricos de la IL-6 y la IL-8, que se consideran involucradas en el dolor, fatiga y depresión de los pacientes. Los autores argumentan que dichas citocinas pudieran ser las encargadas de la modulación de la sintomatología de la enfermedad. ${ }^{11}$ La obesidad tiene efectos sobre la calidad de vida de las personas con padecimientos que provocan dolor crónico. Un IMC elevado se relaciona con un aumento del dolor crónico, discapacidad, depresión y una función física reducida. ${ }^{12,13}$

Durante los últimos años se ha investigado la relación entre la fibromialgia y la obesidad. ${ }^{14}$ Parece que el exceso de peso y grasa juegan un papel importante en dicha enfermedad. La obesidad se ha asociado con una mayor percepción del dolor al presionar puntos sensibles, una disminución de la fuerza, la pérdida de flexibilidad y trastornos del sueño. Estudios han concluido que la obesidad aumenta la gravedad de los síntomas de esta enfermedad. ${ }^{12,15}$

En un estudio, los grupos con IMC más altos presentan una sintomatología de fibromialgia más severa y un deterioro funcional mayor, lo que repercute directamente en la calidad de vida de los enfermos. ${ }^{16}$ De igual forma, se ha demostrado que presentan más comorbilidades médicas, consumen mayor cantidad de medicamentos y realizan menor actividad física, lo cual condiciona la capacidad de efectuar sus ocupaciones cotidianas. ${ }^{17}$ En otro estudio, se concluye que el dolor corporal es mayor en pacientes obesos con fibromialgia comparado con aquellos que presentan un peso normal. ${ }^{18}$

Sin embargo, también existe evidencia en la que no se ha podido asociar el dolor y los síntomas de la fibromialgia con el IMC. ${ }^{19,20}$ Por lo anterior, el objetivo de la presente investigación es describir la asociación de la composición corporal y la sintomatología de fibromialgia.

\section{MATERIALES Y MÉTODOS}

\section{Selección de la muestra}

Se realizó un estudio descriptivo transversal, en el que participaron 27 mujeres del estado de Aguascalientes, México, con diagnóstico de fibromialgia. Las mujeres que participaron contaban con el diagnóstico previo establecido según los criterios del American College of Rheumatology (ACR), por medio de la 
prueba de sensibilidad al dolor (19 partes del cuerpo), además de los apartados anteriormente mencionados. El diagnóstico de cada paciente fue dado por su médico reumatólogo. Todas las participantes recibían tratamiento médico farmacológico para la fibromialgia. ${ }^{2}$

\section{Instrumentos de recolección de información}

La recolección de datos se realizó en tres etapas: 1. Encuesta de datos de la paciente. 2. Evaluación de parámetros antropométricos. 3. Cuestionario sobre los efectos de la fibromialgia (FIQ).

Encuesta de datos personales. La encuesta fue diseñada por los investigadores para obtener información personal sobre las participantes. Incluía: datos personales (nombre, edad y ocupación); antecedentes personales patológicos (diagnóstico médico de la fibromialgia y enfermedades adicionales, fecha de diagnóstico y consumo de medicamentos/tratamiento médico), y datos antropométricos (registro del resultado del análisis de composición corporal).

Evaluación de parámetros antropométricos. El instrumento utilizado fue una báscula de control corporal OMRON HBF-514C para analizar la composición corporal mediante bioimpedancia bioeléctrica, el cual desglosa el peso corporal total en distintos compartimientos como son grasa visceral, grasa corporal total, masa muscular e índice de masa corporal (IMC).

El IMC se define como la relación entre el peso corporal total entre la estatura al cuadrado $(\mathrm{kg} / \mathrm{m} 2)$. Se utilizaron dos criterios para clasificarlo dependiendo de la edad: adultas mayores y adultas jóvenes. La Organización Mundial de la Salud (OMS) clasifica a adultas jóvenes en peso normal (IMC 18.5-24.9kg/ $\mathrm{m} 2$ ), sobrepeso (IMC $25-29.9 \mathrm{~kg} / \mathrm{m} 2$ ) y obesidad (IMC $>40 \mathrm{~kg} / \mathrm{m} 2$ ). Para las adultas mayores clasifica en peso normal (IMC 22-26.9kg/m2), sobrepeso (IMC $27-29.9 \mathrm{~kg} / \mathrm{m} 2)$ y obesidad $(\mathrm{IMC}>30 \mathrm{~kg} / \mathrm{m} 2){ }^{21}$

El porcentaje de grasa es la cantidad de tejido adiposo acumulado en el cuerpo. Gallagher et al. clasifican el porcentaje de grasa en adultas jóvenes en normal (21-33.9), elevado (34-39.9\%) y muy elevado ( $\geq 40 \%)$; para adultas mayores, $24-35.9 \%, 36-41.9 \%$ y $\geq 42$, respectivamente. ${ }^{22}$

El porcentaje de masa muscular y la grasa visceral no fueron categorizados.

Cuestionario sobre los efectos de la fibromialgia (FIQ). Es un instrumento para medir el estado de salud y la calidad de vida relacionada con la salud de personas con fibromialgia. Mide la función física, asuntos laborales (incapacidad laboral y días de incapacidad), depresión, ansiedad, cansancio matutino, fatiga y sensación de bienestar en las semanas previas. Evalúa el impacto de la fibromialgia en 10 dimensiones: capacidad física, trabajo habitual, afectación de la actividad laboral remunerada, dolor, fatiga, sensación de cansancio, rigidez, sensación de bienestar, ansiedad y depresión. Con ellas se genera un puntaje total entre 0 (menor afectación) y 100 (peor resultado); este puntaje lo nombramos FIQTotal.

Las puntuaciones fueron calculadas siguiendo el instructivo oficial: FIQ Fibromyalgia Impact Questionnaire Version 2.1 Scaling and Scoring Version 1.0: August 2011. 
Para analizar los datos se dividió el cuestionario en tres categorías, las cuales son FIQ-1 (nivel de actividad), FIQ-2 (impacto global) y FIQ-3 (intensidad de los síntomas).

La categoría FIQ-1 (nivel de actividad) evalúa el funcionamiento físico con preguntas que estiman la capacidad de realizar actividades cotidianas. La categoría FIQ-2 (impacto global) habla sobre bienestar general en los últimos siete días. Y la categoría FIQ-3 (intensidad de los síntomas) utiliza una escala visual analógica de $10 \mathrm{~cm}$ en la que el paciente califica de la manera que mejor describa cómo se ha sentido en los últimos días, según las siguientes categorías: dolor, dificultad para trabajar, fatiga, cansancio, rigidez, ansiedad y depresión.

Dichas categorías generan un puntaje para dar la clasificación de los efectos de la fibromialgia: leve, moderado o severo, lo cual describe la manera en la que impactan los efectos y síntomas de la fibromialgia en el estado de salud y la calidad de vida.

Para el análisis estadístico se utilizaron los puntajes categorizados de FIQ-1, FIQ-2, FIQ-3 y el FIQ-Total (que considera el puntaje total del cuestionario). ${ }^{23}$

\section{Análisis estadistico}

Los datos del Cuestionario sobre los efectos de la fibromialgia y la composición corporal fueron analizados por el programa estadístico para Windows SPSS versión 24 de la marca IBM, considerando un valor de $\mathrm{p}<0.05$ como estadísticamente significativo. Se utilizó la prueba de Shapiro Wilks para determinar normalidad. Posteriormente, las pruebas usadas para análisis de datos fueron correlación de Spearman y Kruskal Wallis para diferencias.

\section{Consideraciones bioéticas}

En el estudio participaron voluntariamente 27 mujeres de dos instituciones del estado de Aguascalientes, en apego a la declaración de Helsinki y al reglamento de la Ley General de Salud en Materia de la Investigación para la Salud. A todas las participantes se les solicitó consentimiento informado.

\section{RESULTADOS}

\section{Características generales de la población estudiada}

El estudio fue completado con una muestra de 27 mujeres, la media de edad fue de 52.4 \pm 11.3 . En la tabla 1 se pueden observar las características de la población. 
Tabla 1.

Características de las mujeres con fibromialgia

\begin{tabular}{lc}
\hline & Mujeres con fibromialgia (n=27) \\
& \\
Edad (años) & $52.4 \pm 11.3$ \\
Peso (kg) & $72.4 \pm 15.7$ \\
Talla (m) & $1.58 \pm 0.06$ \\
$\mathrm{IMC}\left(\mathrm{kg} / \mathrm{m}^{2}\right)$ & $28.9 \pm 5.4$ \\
\hline Composición corporal & $42.3 \pm 6.7$ \\
\hline Masa grasa (\%) & $24.1 \pm 2.2$ \\
Masa muscular (\%) & $9 \pm 2.3$ \\
Grasa visceral & \\
IMC: Índice de masa corporal. Datos representados en media y desviación \\
estándar o mediana v rangos intercuartiles de acuerdo con su distribución.
\end{tabular}

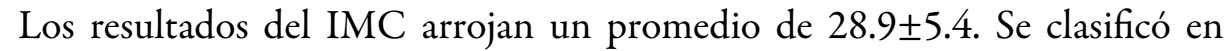
desnutrición, normopeso, sobrepeso y obesidad generando porcentajes de $3.7 \%$, $25.9 \%, 29.6 \%$ y $40.7 \%$, respectivamente (figura 1 ).

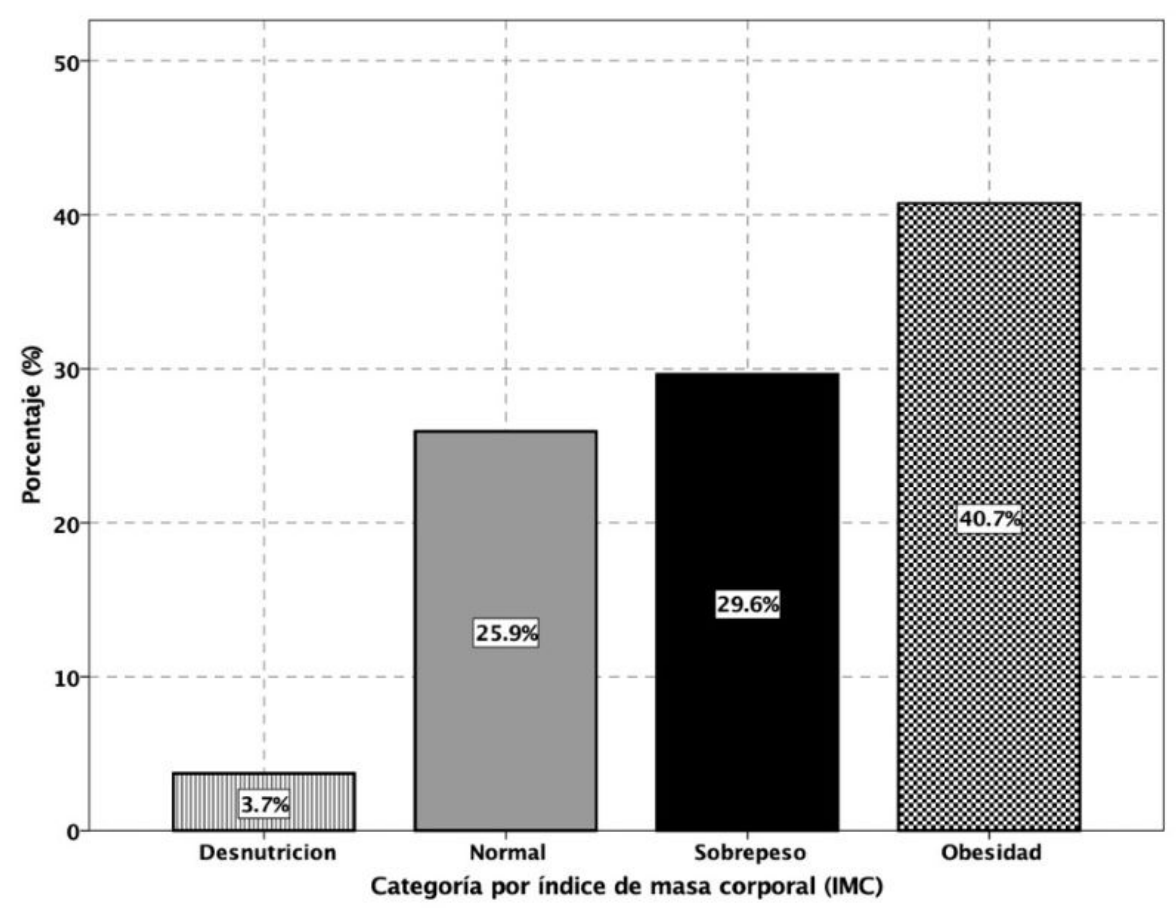

Figura 1.

Índice de masa corporal de las mujeres con fibromialgia

El 66.7\% de las pacientes presentó porcentaje muy elevado de grasa, el 22.9\% obtuvo porcentaje elevado y solo el $11.1 \%$ presento un porcentaje normal para su edad.

Los grupos de medicamentos que mayormente consume la población estudiada son los antiinflamatorios no esteroideos (AINES) con un porcentaje 
de $30 \%$; seguido de antiepilépticos, con un $28 \%$, y, por último, antidepresivos y analgésicos, con un $21 \%$.

Además de la fibromialgia, las enfermedades más frecuentes fueron hipertensión (27\%), colitis (22\%), gastritis (17\%) y diabetes mellitus (12\%).

\section{Diferencias de la sintomatología de la fibromialgia según su composición corporal}

En los resultados arrojados por el FIQ, se encuentra que el $48.1 \%$ de las mujeres con fibromialgia presentaron moderada intensidad de síntomas, el $37.1 \%$ severa intensidad y el $14.8 \%$ leve intensidad (figura 2).

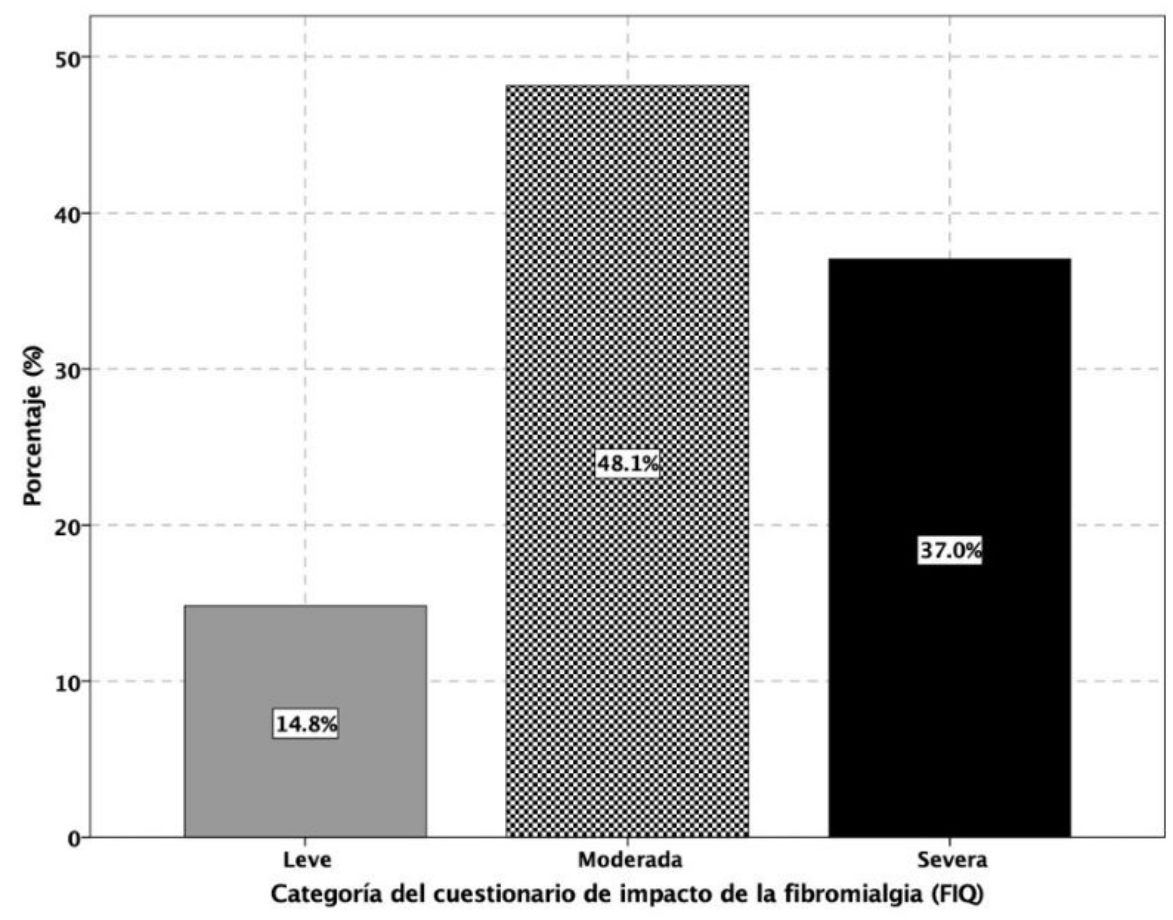

Figura 2.

Prevalencia de síntomas clasificados de acuerdoal cuestionario de impacto de la fibromialgia (FIQ)

En la figura 3 se puede observar que las personas con obesidad presentaron un menor puntaje total del FIQ; sin embargo, dicha diferencia no fue significativa. Asimismo, en las categorías del cuestionario tampoco se encontró diferencia significativa. 

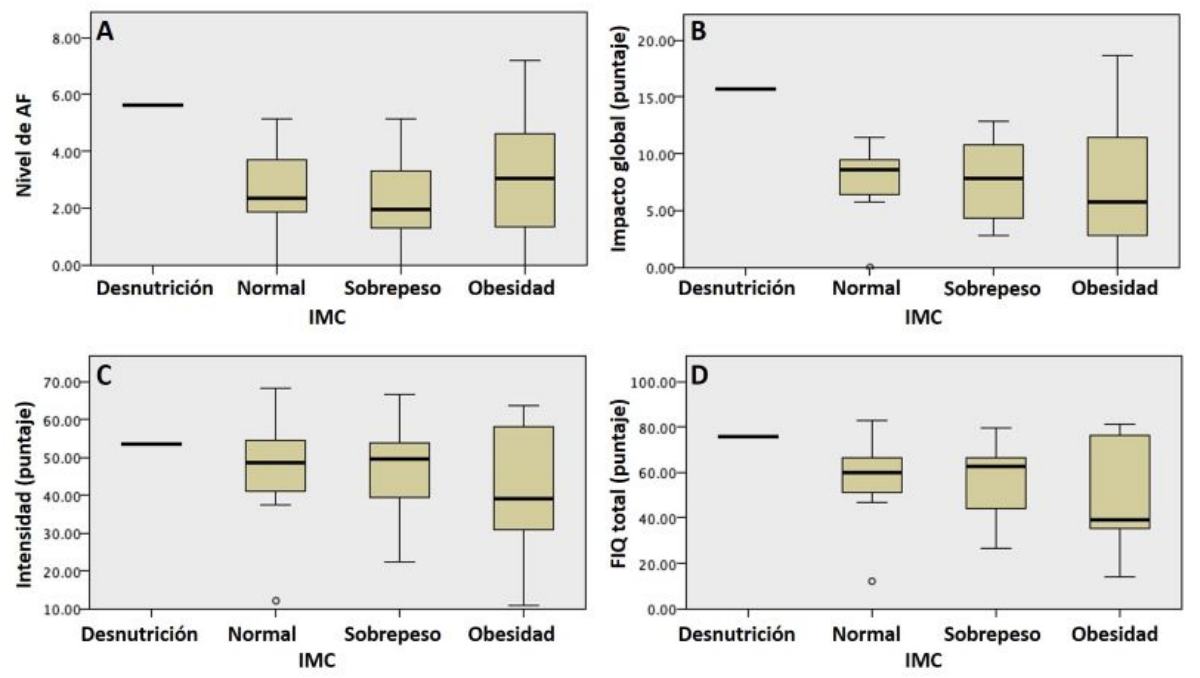

Figura 3.

A) Nivel de actividad física de acuerdo al IMC, B) Puntaje del impacto global de acuerdo al IMC, C) Severidad de sintomatología (puntaje de intensidad) de acuerdo al IMC y D) Puntaje total del FIQ de acuerdo al IMC.

Fuente: Realización propia. IMC: Índice de masa corporal; FIQ: Cuestionario de impacto de la fibromialgia.

No hubo diferencias entre un porcentaje de grasa normal, elevado y muy elevado en relación con el puntaje total del FIQ (figura 4).
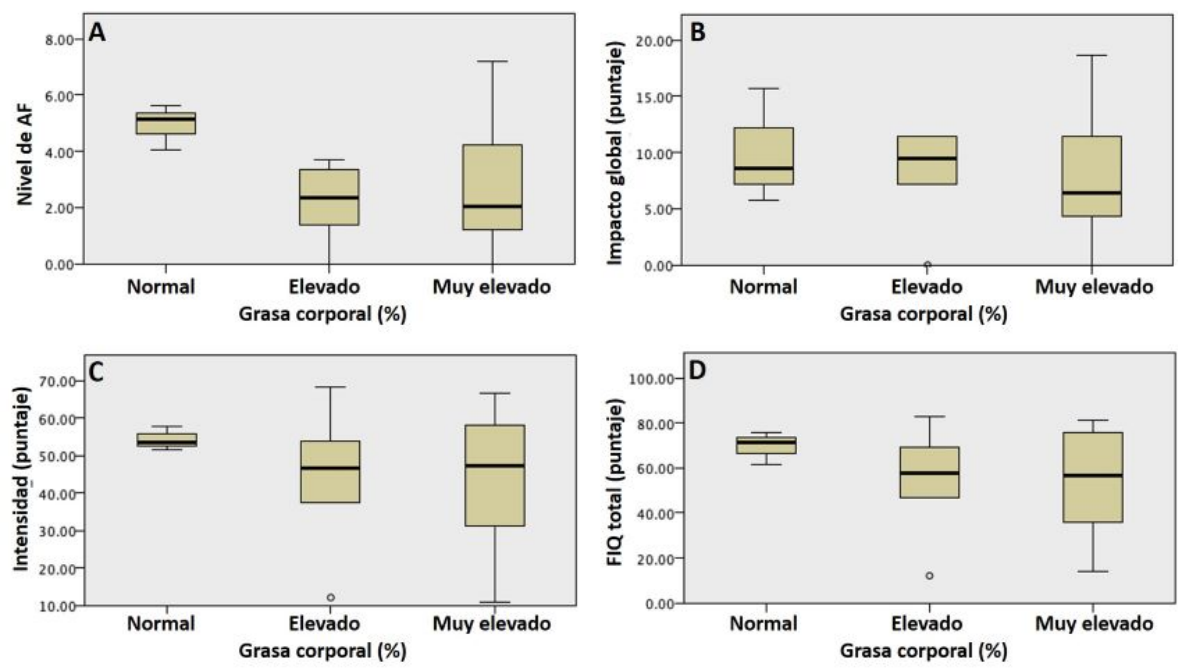

Figura 4.

A) Nivel de actividad física de acuerdo a la grasa corporal, B) Puntaje del impacto global de acuerdo a la grasa corporal, C) Severidad de sintomatología (puntaje de intensidad) de acuerdo a la grasa corporaly D) Puntaje total del FIQ de acuerdo a la grasa corporal.

Fuente: Realización propia. AF: Actividad física; FIQ: Cuestionario de impacto de la fibromialgia.

El puntaje total del FIQ en relación con la grasa visceral y el porcentaje de músculo no fue estadísticamente significativo, presentando valores de $\mathrm{p}=.620 \mathrm{y}$ $\mathrm{p}=.851$, respectivamente. 


\section{Relación entre la composición corporal y las subcategorias del FIQ}

La tabla 2 muestra la relación entre la composición corporal y las variables clínicas obtenidas en el FIQ, demostrando que no hay relación significativa.

Tabla 2.

Asociación de composición corporal y FIQ en mujeres con fibromialgia

\begin{tabular}{l|c|c|c|c|c}
\hline & Peso & IMC & $\begin{array}{c}\% \text { Grasa } \\
\text { corporal }\end{array}$ & $\begin{array}{c}\text { \% Masa } \\
\text { muscular }\end{array}$ & $\begin{array}{c}\text { Grasa } \\
\text { visceral }\end{array}$ \\
\hline & $\begin{array}{c}\text { Valor de } \mathrm{p} \\
(\mathrm{r})\end{array}$ & $\begin{array}{c}\text { Valor de } \mathrm{p} \\
(\mathrm{r})\end{array}$ & $\begin{array}{c}\text { Valor de } \mathrm{p} \\
(\mathrm{r})\end{array}$ & $\begin{array}{c}\text { Valor de } \mathrm{p}(\mathrm{r}) \\
\text { Valor de } \mathrm{p} \\
(\mathrm{r})\end{array}$ \\
\hline FIQ-1 & $.475(-.143)$ & $.755(-.063)$ & $.718(-.073)$ & $.656(.090)$ & $.744(-.066)$ \\
\hline FIQ-2 & $.443(-.154)$ & $.590(-.109)$ & $.744(-.066)$ & $.981(-.005)$ & $.806(-.050)$ \\
\hline FIQ-3 & $.350(-.187)$ & $.743(-.066)$ & $.879(-.0 .31)$ & $.916(-.021)$ & $.845(.039)$ \\
\hline Total FIQ & $.442(-.155)$ & $.806(-.049)$ & $.980(-.005)$ & $.787(-.055)$ & $.861(.035)$ \\
\hline
\end{tabular}

IMC: Índice de masa corporal. FIQ: Cuestionario sobre los efectos de la fibromialgia. FIQ-1: Nivel de actividad. FIQ-2: Impacto global. FIQ-3: Intensidad de los síntomas. Prueba estadística utilizada: correlación de Spearman

\section{DISCUSIÓN}

En la actualidad, la obesidad es un padecimiento muy frecuente, y se le ha visto relacionado con diversas enfermedades. Se ha observado, asimismo, un peor pronóstico en aquellas personas que presentan obesidad que en quienes no la padecen.

En esta investigación, se estudió la asociación entre la sintomatología de la fibromialgia y la composición corporal, tomando en cuenta parámetros antropométricos como el IMC, además del porcentaje de grasa y músculo en población femenina con diagnóstico de fibromialgia.

Se encontró que la prevalencia de sobrepeso y obesidad en pacientes con fibromialgia fue del $29.6 \%$ y $40.7 \%$ respectivamente, lo que coincide con otros estudios en los que la prevalencia de obesidad fue de 32 al $50 \%$ y la de sobrepeso fue del 21 a $30 \% .5,6,7 \mathrm{El}$ IMC promedio fue de $28.9 \mathrm{~kg} / \mathrm{m} 2$, similar al que encontró Correa Rodríguez et al., en el que se muestra una media de $29.11 \mathrm{~kg} /$ m. ${ }^{2,24}$

Por otro lado, el grupo de medicamentos de mayor consumo en la muestra estudiada fue el de los antiinflamatorios no esteroideos (AINES). Como bien se sabe, este tipo de medicamentos resulta útil en la disminución del dolor periférico o inflamación. En un estudio, se informó que aún no existe suficiente evidencia sobre los beneficios de los AINES; sin embargo, se sabe que son los fármacos más recomendados para tratar la enfermedad. Se menciona que, probablemente, es debido a su uso de dosis subterapéuticas, en las que se comporta como analgésico. $^{25}$ 
Se ha comprobado que los fármacos antidepresivos posen capacidad analgésica propia y mejoran otras manifestaciones clínicas de los pacientes incluyendo el sueño, la fatiga, el dolor y el estado de ánimo. En un estudio, se comparó la eficacia de la amitriptilina y la nortriptilina contra un placebo en 118 pacientes con fibromialgia durante dos meses de tratamiento. Se obtuvieron resultados que mostraron una mejoría significativa, tanto en capacidad funcional, como en la escala visual análoga del dolor en aquellas pacientes que tomaban algún antidepresivo. Cabe mencionar que no se encontró diferencia significativa en los efectos entre ambos fármacos. ${ }^{25}$

Es importante señalar que, en la presente investigación, las pacientes estaban controladas con tratamiento farmacológico para disminuir la sintomatología. A pesar de lo dicho, en los datos arrojados por el FIQ se encontró que el $48.1 \%$ refería una moderada intensidad de la sintomatología, aun siendo controladas por agentes farmacológicos, lo que podría estar relacionado con el sobrepeso y la obesidad; sin embargo, aún no existe evidencia sufriente. ${ }^{25}$

No se encontraron diferencias significativas entre los rangos del IMC, la clasificación del porcentaje de grasa y el puntaje de las subcategorías y el total del FIQ, contrario a lo que encontró Chul-Hyun Kim et al., donde se obtuvo que los grupos de IMC más altos presentaron una sintomatología más severa y un deterioro funcional mayor. ${ }^{16}$ Este último hallazgo coincide con lo que Kamal M. y cols. encontraron en un estudio realizado con 75 mujeres y ocho hombres con diagnóstico de fibromialgia y obesidad, en donde se observó menor sintomatología evaluada mediante el FIQ al disminuir de peso. ${ }^{5}$ Una investigación más encontró mayor sensibilidad al dolor en pacientes con obesidad. ${ }^{16}$ Sin embargo, la evidencia sigue siendo controversial, ya que en el estudio de Yunus M.B. et al., que fue realizado en 211 pacientes con fibromialgia, no se encontró asociación significativa entre el IMC y el dolor. ${ }^{19}$ Del mismo modo, Gota C.E. et al., no encontraron asociación entre IMC y los síntomas de la fibromialgia, los cuales fueron medidos por el FIQ, lo que coincide con lo obtenido en la presente investigación. ${ }^{17}$

Una posible explicación de que nuestros resultados no fueron significativos sería que solo se usó un instrumento para la evaluación de la sintomatología (FIQ), en comparación con otros estudios en los cuales se utilizó un instrumento para evaluar cada uno de los síntomas por separado (ansiedad, calidad del sueño, fatiga y depresión). ${ }^{16,24}$ Sin embargo, es importante mencionar que el FIQ es uno de los instrumentos más utilizados y recomendados para medir el impacto de los síntomas de la fibromialgia.

Una de las limitantes de la presente investigación fue que la muestra se eligió a conveniencia y se completó con 27 pacientes. Mientras que en otros artículos revisados el tamaño de la muestra fue mayor. En un estudio hecho en 205 pacientes con fibromialgia, se encontró que la obesidad puede complicar el cuadro clínico, aunque se incita a otros autores a seguir investigando sobre la mejoría en la fibromialgia debido a la reducción de peso. ${ }^{15,24}$

La fibromialgia puede manifestarse a cualquier edad y se presenta de manera similar en diferentes países y culturas; sin embargo, la edad en la que dicha enfermedad genera más impacto es entre los 25 y los 50 años. ${ }^{1}$ Algo para tomar en cuenta es que, en la presente investigación, los rangos de edad fueron muy 
variados, encontrándose entre 23 y 71 años. Uno de los cambios fisiológicos que se sufre a lo largo de la vida es la modificación en la composición corporal, presentándose una disminución en la fuerza y tono muscular a la par que aumenta la masa de grasa. A dicho cambio lo conocemos como sarcopenia. ${ }^{26}$ Para futuras investigaciones, se sugiere que los rangos de edades de la muestra no sean tan amplios, para poder tener un mayor control de las variables fisiológicas.

Similar a nuestro estudio, existen otros cuantos en los cuáles la asociación entre el IMC o la composición corporal y la severidad de los síntomas de fibromialgia no pudo ser encontrada. Incluso los estudios en los que dicha asociación existe, se argumenta que sus descubrimientos pueden estar sesgados, debido a que no se utilizaron las herramientas o cuestionarios más adecuados para medir la intensidad de los síntomas, no se presenta el número de participantes suficientes o los resultados no pueden ser extrapolables por el modo de selección de la muestra, entre otras debilidades. Por esa razón, no existe suficiente evidencia para llegar a una conclusión. Sin embargo, es importante tener en cuenta que existen diversos factores que debemos considerar y que pueden explicar la asociación entre fibromialgia y obesidad, entre ellos están una peor calidad del sueño, un mayor nivel de receptores de dolor en el tejido adiposo, y citocinas proinflamatorias elevadas en suero, disfunción autonómica. De igual forma, vale la pena recordar que existe evidencia de que los síntomas musculoesqueléticos, la discapacidad funcional, la calidad de vida, los trastornos psicológicos y, en general, los síntomas de la fibromialgia mejoran con medidas de reducción de peso. $12,16,17,24,27,28$

Como lo menciona Correa en su estudio, la relación entre la obesidad y el dolor crónico no es directa, esta depende de la modulación de diversos factores, tales como los mediadores inflamatorios, cambios estructurales asociados con la obesidad y las características del estilo de vida. De este modo, esta podría ser una de las razones de porqué no se encontró una relación significativa en esta investigación, ya que varios factores que interactúan entre sí (como la edad de las pacientes, etapas fisiológicas como la menopausia, el consumo de medicamentos, el apego al tratamiento y otras enfermedades que presentaban las mujeres) podrían haber impactado los resultados de nuestra investigación. ${ }^{24}$

Aunque la relación no sea clara, la alta prevalencia de obesidad en pacientes con fibromialgia es una razón suficiente para que se siga estudiando y así poder otorgar un tratamiento adecuado para que los pacientes con fibromialgia y obesidad tengan una mejor calidad de vida.

\section{CONCLUSIÓN}

No se encontró relación significativa entre la composición corporal y la sintomatología de fibromialgia, sin embargo, existe evidencia que demuestra lo contrario. Dado que la prevalencia de obesidad y sobrepeso es alta en pacientes con fibromialgia, es importante seguir investigando para establecer la relación que existe entre estos dos padecimientos y así poder otorgar un adecuado tratamiento integral. 


\section{REFERENCIAS}

1. Guzmán-Silahua S, Muñoz-Gaytán D, Medoza-Vázquez G, Orozco-Boracio G, Rodríguez-Ruiz J, García-de la Torre I, et al. Fibromialgia. El residente, 2018; 13(2):62-67.

2. Covarrubias-Gómez A., Carrillo-Torres O. Actualidades conceptuales sobre fibromialgia. Revista Mexicana de Anestesiología, 2016; (39):58-63.

3. Farreras V., Rozman C. Medicina Interna. 17a edición. Barcelona, España: Elsevier; 2012.

4. Pasquel A., De Sousa A, Berssaneti A., Akemi M., Lee S. La prevalencia de la fibromialgia: revisión de la literatura actualizada. Rev bras reumatol, 2017; 57(4):356-363.

5. Kamal M, Sallam R, Salah H, Elarman M. Effect of weight reduction on the quality of life in obese patients with fibromyalgia syndrome: a randomized controlled trial. Clin rheumatol, 2012; 31(1):1591-1597.

6. De Araújo T.A., Mota M.C., Crispim C.A. Obesity and sleepiness in women with fibromyalgia. Rheumatology International, 2014; 35(2):281-287.

7. Instituto Nacional de Salud Pública. Encuesta de salud y nutrición 2012. [citado el: 19 de septiembre de 2019]. Disponible en: https://ensanut.insp.mx/encuestas/ ensanut2012/doctos/informes/ENSANUT2012ResultadosNacionales.pdf

8. Blancas-Flores G., Almanza-Pérez J.C., López-Roa RI, et al. La obesidad como un proceso inflamatorio. Bol Med Hosp Infant Mex. 2010;67(2):88-97.

9. Sánchez F., García R., Alarcón F., Cruz M. Adipocinas, Tejido adiposo y su relación con células del sistema inmune. Gac.Med Mex, 2005; 141(6):505-512.

10. Wallace D.J., Linker-Israeli M., Hallegua D., Silverman S., Silver D., Weisman M.H. Cytokines play an aetiopathogenetic role in fibromyalgia: a hypothesis and pilot study. Rheumatology (Oxford). 2001 Jul;40(7):743-9. doi: 10.1093/ rheumatology/40.7.743. PMID: 11477278.

11. Moreno R., Mico J. TNF y citocinas y dolor: más allá de la inflamación tisular. Reumatol. Clinic, 2009; 5(2):1-4.

12. Arranz L.I., Rafecas M., Alegre C. Effects of Obesity on Function and Quality of Life in Chronic Pain Conditions. Curr Rheumatol Rep, 2014; (16):390-397.

13. Marcus DA. Obesity and the impact of chronic pain. Clin J Pain, 2004; 20(3):18691.

14. Ursini F, Naty S, Grembiale R. Fibromyalgia and obesity: the hidden link. Rheumatol Int, 2011; 31(1):1403-1408.

15. Okifuji A, Donaldson GW, Barck L, et al. Relationship between fibromyalgia and obesity in pain, function, mood, and sleep. J Pain, 2010; 11(12):1329-37.

16. Kim C-H, Luedtke C, Vincent A, Thompson J, Terry A. Association of Body Mass Index with symptom severity and quality of life in patients with fibromyalgia. Arthritis Care \& Research, 2012; 64(2):222-228.

17. Gota C, Kaouk S, Wilke W. The association between Body Mass Index and disability, depression, history of abuse, medications, and comorbidities. JCR: Journal of Clinical Rheumatology, 2015; 21(6):289-295.

18. Aparicio V, Ortega F, Carbonell-Baeza A, Camilettia D, Ruiza J, DelgadoFernández M. Relationship of Weight Status with Mental and Physical Health in Female Fibromyalgia Patients. Obes Facts, 2011; 4(1):443-448. 
19. Yunus MB, Arslan S, Aldag JC. Relationship between body mass index and fibromyalgia features. Scand J Rheumatol, 2002; (31):27-31.

20. Gota C, Kaouk S, Wilke W. The association between Body Mass Index and disability, depression, history of abuse, medications, and comorbidities. JCR: Journal of Clinical Rheumatology, 2015; 21(6):289-295.

21. Organización Mundial de la Salud. Obesidad y Sobrepeso. Organización Mundial de la Salud. [citado el 22 de enero del 2020]. Disponible en: https://www.who.int/ es/news-room/fact-sheets/detail/obesity-and-overweight

22. Gallagher. Interpretación de resultados del porcentaje de grasa corporal. American Journal of Clinical Nutrition, 2000; (72):10-11.

23. Burckhardt CS, Clark SR, Bennett RM. El cuestionario de impacto de fibromialgia: Desarrollo y validación. J Rheumatol, 1991; 18(5):728-733.

24. Correa-Rodríguez M, Mansouri-Yachou JE, Casas-Barragan A, Molina F, RuedaMedina B, Aguilar-Ferrándiz ME. The Association of Body Mass Index and Body Composition with Pain, Disease Activity, Fatigue, Sleep and Anxiety in Women with Fibromialgy. Nutrients, 2019; (11):1-13.

25. Rivera J. Tratamiento Farmacológico en la Fibromialgia. Semin Fund Española Reumatol, 2011; (1):21-26.

26. Fielding S. Sarcopenia: An undiagnosed condition in older adults. Current consensus definition: prevalence, etiology, and consequences; J Am Med Dir Assoc, 2011; (12):249-256.

27. Senna MK, Sallam RA-ER, Ashour HS, Elarman M. Effect of weight reduction on the quality of life in obese patients with fibromyalgia syndrome: a randomized controlled trial, Clinical Rheumatology, 2012; 31(11):1591-1597.

28. Shapiro JR, Anderson DA, Danoff-Burg S. A pilot study of the effects of behavioral weight loss treatment on fibromyalgia symptoms. J Psychosom Res, 2005; (59):275-82. 